\title{
Anti Error Propagation Methods for Wireless Uplink Using Network Coding
}

\author{
Wei Guan and K. J. Ray Liu \\ Department of Electrical and Computer Engineering \\ University of Maryland, College Park, MD 20742 \\ Email: \{wguan, kjrliu\}@umd.edu
}

\begin{abstract}
Wireless network coding suffers the error propagation issues that may severely degrade the diversity performance. In this work, we develop two power scaling schemes at the relay side and two detection schemes at the receiver side, respectively, to mitigate error propagation in network-coded uplink channel and thus achieve full diversity. For the soft power scaling based link adaptive relaying, we develop a virtual channel model and demonstrate that the relay power should be such to balance the signal-to-noise ratios of the source-relay channel and relaydestination channel. As for the hard power scaling based $\mathrm{ON}$ OFF relaying, we first design a decision rule based on total pairwise error probability, and then simplifies it to the thresholdbased relaying strategy. At the receiver side, we show that the weighted minimum distance detection with the weight being determined by the relative link quality of source-relay channel and relay-destination channel can achieve full diversity once the global channel state information is available, otherwise the maximum likelihood detection that explicitly takes into account relay decoding error should be employed to achieve full diversity.
\end{abstract}

\section{INTRODUCTION}

Cooperative communications have gained much attention recently due to its potential to provide spatial diversity, extend transmission coverage and save power consumption [1]. However, the conventional repetition coding based relaying protocols such as decode-and-forward (DF) and amplify-andforward (AF) [2] have the shortcomings of low spectral efficiency. So network coding has been proposed to address this issue.

Compared to its wired counterpart, wireless network coding suffers severe random decoding error due to channel fading [3][4]. In practice, strong error detection/correction methods like cyclic redundancy check (CRC) could be exploited to prevent error propagation [5]. But the cost in terms of lower bandwidth efficiency and larger decoding delay becomes formidable in many applications. Consequently, mitigating error propagation through smart design of relaying and detection schemes is gaining more and more focus recently. For example, it is reported in [6]-[7] that threshold-based ONOFF relaying can achieve full diversity order for DF relays. Instead of hard power scaling, [8] proposes a soft power scaling protocol based on the relative link quality of sourcerelay channel and relay-destination channel. At the receiver side, cooperative maximum-ratio combining [9] and maximum likelihood detector [10] are advocated to achieve full diversity.

We note that the above-mentioned methods [6]-[10] are mainly proposed for the repetition coding based DF relaying system which only involves a single source-destination pair. Consequently, these schemes are not directly applicable for a general multi-user network-coded system, which is potentially more vulnerable to relay decoding error. In a limited number of literatures discussing error control in network-coded networks, [11] and [12] extend the weighted combining technique [9] and maximum likelihood detection (MLD) [10], respectively. However, global channel state information (CSI) needs to be known at the receiver, which greatly increases the signaling overhead. MLD based on local CSI is investigated in [13], but the channel fading effects are neglected to simplify the analysis. Another common weakness of the above work [11][13] is that only the receiver-side technique is considered. In the cellular systems, the user devices generally have very limited power and processing capability. Consequently these complicated strategies may not be applicable in such scenarios.

We also remark that in many literatures focusing on network coding design (e.g., [5][14]), it is assumed that the decoding error can be perfectly detected/corrected, which is impractical in real systems. Indeed, when there does exist relay decoding error, the diversity performance of the network-coded system may severely degrade. So investigating efficient schemes to control error propagation in a multi-user network-coded system is an important issue that motivates the current work. To be specific, we consider the wireless uplink channel where a single relay node helps the two source nodes send messages to a single destination by use of the network coding. We first analyze the pairwise error probability (PEP) for binary phase-shift keying (BPSK) signal, and show that the dominant error event occurs when there is only one incorrectly decoded symbol at the destination. We then develop the soft/hard power scaling schemes at the relay side, and the weighted minimum distance detection (MDD) and MLD at the receiver side, respectively, to achieve full diversity. We perform extensive simulations to verify our analysis.

Notations: We use abbreviation i.i.d. for independent and identically distributed, and denote $Z \sim \mathcal{C N}\left(\mu, \sigma^{2}\right)$ as a circularly symmetric complex Gaussian random variable $Z$ with i.i.d. real part and imaginary part $\sim \mathcal{N}\left(\mu, \frac{\sigma^{2}}{2}\right)$. The probability of an event $\mathcal{A}$ and the probability density function (PDF) of a random variable $Z$ are denoted by $\operatorname{Pr}(\mathcal{A})$ and $f(Z)$, respectively. We define $Q(x)=\frac{1}{\sqrt{2 \pi}} \int_{x}^{\infty} e^{-\frac{t^{2}}{2}} d t$ as the 
Q-function, and denote $g\left(z, \sigma^{2}\right)=\frac{1}{\pi \sigma^{2}} \mathrm{e}^{-\frac{|z|^{2}}{\sigma^{2}}}$ as the PDF of $Z \sim \mathcal{C N}\left(0, \sigma^{2}\right)$. Finally, we say $h(x)=\mathrm{O}(g(x))$ if $\limsup _{x \rightarrow \infty} \frac{h(x)}{g(x)}<\infty$.

\section{SYSTEM MODEL}

Consider a wireless uplink channel with two source nodes sending data to the destination assisted by a single relay node employing network coding. In the $k$ th phase for $k=1,2$, the $k$ th source broadcasts its message to the relay and destination. The received signal can be represented as

$$
y_{k t}=h_{k t} \sqrt{P} x_{k}+n_{k t}=\bar{h}_{k t} x_{k}+n_{k t}
$$

for $t \in\{r, d\}$ and $k=1,2$. Here $y_{k t}$ is the received signal at node $t$ from source $k ; n_{k t} \sim \mathcal{C N}\left(0, N_{0}\right)$ is the additive noise; $h_{k t} \sim \mathcal{C N}\left(0, \lambda_{k t}\right)$ is the rayleigh fading channel coefficient with $\lambda_{k t}$ being the channel gain; $P$ is the transmitted power; and $x_{k}$ is the source symbol with normalized power, i.e., $E\left(\left|x_{k}\right|^{2}\right)=1$. To simplify the analysis, we assume binary phase-shift keying (BPSK) signal is used by the two sources, i.e., $x_{k} \in \Omega=\{1,-1\}$. However, most of our conclusions can be easily extended to any higher-order modulations. For notational convenience, we also define the XORed source symbol as ${ }^{1}$

$$
x_{\oplus}=x_{1} \oplus x_{2}=-x_{1} x_{2} .
$$

Note that $x_{\oplus} \in\{-1,1\}$ is also BPSK signal. Besides, we define $\bar{h}_{k t}=\sqrt{P} h_{k t}$ as the equivalent channel, and define $\gamma_{k t}=\left|h_{k t}\right|^{2} \Gamma$ as the instantaneous channel SNR with $\Gamma=\frac{P}{N_{0}}$ being the reference system SNR. It is easy to show that $\gamma_{k t}$ is an exponential random variable with mean $\Gamma_{k t}=\lambda_{k t} \Gamma$.

As the source symbols are randomly picked from the constellation with equal probability, MLD is equivalent to MDD at the relay node, i.e.,

$$
x_{r, k}=\arg \min _{\hat{x}_{k} \in \Omega}\left|y_{k r}-\bar{h}_{k r} \hat{x}_{k}\right|^{2}
$$

for $k=1,2$. Then, the decoded source messages are mixed through XOR operation, and the re-encoded message is

$$
x_{r, \oplus}=x_{r, 1} \oplus x_{r, 2}=-x_{r, 1} x_{r, 2} .
$$

In the third phase, the relay node shall forward the networkcoded message $x_{r, \oplus}$ to the destination, and the received signal is

$$
y_{r d}=h_{r d} \sqrt{\alpha P} x_{r, \oplus}+n_{r d}=\bar{h}_{r d} \sqrt{\alpha} x_{r, \oplus}+n_{r d} .
$$

Here $n_{r d} \sim \mathcal{C N}\left(0, N_{0}\right)$ is the additive noise, and $h_{r d} \sim$ $C N\left(0, \lambda_{r d}\right)$ is the rayleigh fading channel coefficient with $\lambda_{r d}$ being the channel gain. Besides, we define $\bar{h}_{r d}=\sqrt{P} h_{r d}$ as the equivalent relay-destination channel, and define $\gamma_{r d}=$ $\left|h_{r d}\right|^{2} \Gamma$ as the corresponding channel SNR that follows exponential distribution with mean $\Gamma_{r d}=\lambda_{r d} \Gamma$. Without loss of generality, we assume the additive noises and channel coefficients of different channels are all independent. Note that

\footnotetext{
${ }^{1}$ Note that the conventional XOR operation is performed at bit-level. Here we omit the bit-to-symbol mapping and use the equivalent symbol-leve XORed output directly.
}

the power scaling coefficient $\alpha$ in (5) could be adaptive to channel conditions, as will be clear later.

As there is no error detection/correction code, neither the relay node nor the destination knows the decoding status of $x_{r, \oplus}$, i.e., whether $x_{r, \oplus}=x_{\oplus}$ or not. So weighted MDD can be employed at the destination to jointly decode the two source symbols based on the observations $y_{1 d}, y_{2 d}$ and $y_{r d}$, i.e.,

$$
\begin{aligned}
& \mathbf{x}_{d} \triangleq\left(x_{d, 1}, x_{d, 2}\right) \\
& =\arg \min _{\hat{x}_{1}, \hat{x}_{2} \in \Omega}\left(\sum_{k=1}^{2}\left|y_{k d}-\bar{h}_{k d} \hat{x}_{k}\right|^{2}+w\left|y_{r d}-\bar{h}_{r d} \sqrt{\alpha} \hat{x}_{\oplus}\right|^{2}\right),
\end{aligned}
$$

where the combining weight $w$ can be leveraged to account for the possible relay decoding error, as will be clear later. Our major contribution of this work is to design some special power scaling coefficient $\alpha$ and combining weight $w$ such that the end-to-end error performance achieves full diversity.

We remark that the link adaptive techniques we shall discuss later depends largely on how much CSI is known at each node. For local CSI based methods, we assume that the receiver of each channel knows the corresponding instantaneous channel coefficient (or equivalently, the instantaneous channel SNR). Specifically, $\bar{h}_{k r}\left(\bar{\gamma}_{k r}\right)$ are known at the relay node for $k=1,2$, and/or $\bar{h}_{k d}\left(\gamma_{k d}\right)$ and $\bar{h}_{r d}\left(\gamma_{r d}\right)$ are known at the destination for $k=1,2$. For global CSI based methods, we further assume that the relay node knows $\bar{h}_{r d}\left(\gamma_{r d}\right)$ and/or the destination knows $\bar{h}_{k r}\left(\gamma_{k r}\right)$ for $k=1,2$ besides the above assumptions. As the average channel SNRs are second-order statistics that stay stationary over a long time, we assume that they are available to all nodes with trivial feedback overhead.

\section{GENERAL ERROR PERFORMANCE ANALYSIS}

Before proceeding to discuss the detailed design of relaying and detection schemes, we first analyze the general end-toend error performance. In this work, we are interested in the diversity order of the error performance, which is defined as

$$
d i v=-\log _{\Gamma \rightarrow \infty} \frac{\log \operatorname{Pr}\left(\mathbf{x}_{\mathbf{d}} \neq \mathbf{x}\right)}{\log \Gamma},
$$

where $\mathbf{x}=\left(x_{1}, x_{2}\right)$ is the source symbol vector. Note that the maximum diversity order is 2 because each source symbol can reach the receiver through two independent channels, i.e., the individual direct link and the common relay branch, as the network-coded symbol provides side information for both sources. Unfortunately, the exact error analysis is intractable due to the complexity in deriving the closed-form decision regions of (6). Alternatively, we shall investigate the pairwise error probability (PEP), which is well known as a tight bound of the real error probability.

Using the law of total probability, we can express PEP as

$$
\begin{aligned}
\operatorname{Pr}(\mathbf{x} & \rightarrow \hat{\mathbf{x}})=\operatorname{Pr}\left(\mathbf{x} \rightarrow \hat{\mathbf{x}}, \Phi_{\text {prop }}, \Phi_{\text {on }}\right) \\
& +\operatorname{Pr}\left(\mathbf{x} \rightarrow \hat{\mathbf{x}}, \Phi_{\text {free }}, \Phi_{\text {on }}\right)+\operatorname{Pr}\left(\mathbf{x} \rightarrow \hat{\mathbf{x}}, \Phi_{\text {off }}\right) .
\end{aligned}
$$

Here $\Phi_{o n}$ and $\Phi_{o f f}$ are the events that the relay node does forward the message (i.e., $\alpha \neq 0$ ) and that the relay node stays 
idle (i.e., $\alpha=0$ ), respectively. In the case of $\alpha=0$, the weight $w$ in (6) should be set to 0 too as there is no information sent from the relay node at all. On the other hand, $\Phi_{\text {free }}$ is the event that the relay node obtains the correct network-coded symbol (i.e., $x_{r, \oplus}=x_{\oplus}$ ), and $\Phi_{\text {prop }}$ means $x_{r, \oplus} \neq x_{\oplus}$ and the relay decoding error may propagate to the destination. Accordingly, we have

$$
\begin{aligned}
\operatorname{Pr}\left(\Phi_{\text {prop }}\right) & =\frac{1}{2}\left(1-\sqrt{\frac{\Gamma_{1 r}}{1+\Gamma_{1 r}}} \sqrt{\frac{\Gamma_{2 r}}{1+\Gamma_{2 r}}}\right) \\
\Gamma & \underset{\approx}{\approx} \frac{\lambda_{1 r}+\lambda_{2 r}}{4 \lambda_{1 r} \lambda_{2 r}} \Gamma^{-1},
\end{aligned}
$$

and $\operatorname{Pr}\left(\Phi_{\text {free }}\right)=1-\operatorname{Pr}\left(\Phi_{\text {prop }}\right)$.

After some manipulations, it is also straightforward to obtain the conditional PEPs as ${ }^{2}$

$$
\left\{\begin{array}{c}
\operatorname{Pr}(\mathbf{x} \rightarrow-\mathbf{x} \mid h)=Q\left(\sqrt{2 \sum_{k \in\{1,2\}} \gamma_{k d}}\right) \\
\operatorname{Pr}\left(\mathbf{x} \rightarrow\left(-x_{1}, x_{2}\right) \mid \Phi_{o f f}, h\right)=Q\left(\sqrt{2 \gamma_{1 d}}\right) \\
\operatorname{Pr}\left(\mathbf{x} \rightarrow\left(-x_{1}, x_{2}\right) \mid \Phi_{\text {free }}, \Phi_{o n}, h\right) \\
=Q\left(\frac{\sqrt{2}\left(\gamma_{1 d}+\alpha w \gamma_{r d}\right)}{\sqrt{\gamma_{1 d}+\alpha w^{2} \gamma_{r d}}}\right) \\
\operatorname{Pr}\left(\mathbf{x} \rightarrow\left(-x_{1}, x_{2}\right) \mid \Phi_{\text {prop }}, \Phi_{o n}, h\right) \\
=Q\left(\frac{\sqrt{2}\left(\gamma_{1 d}-\alpha w \gamma_{r d}\right)}{\sqrt{\gamma_{1 d}+\alpha w^{2} \gamma_{r d}}}\right)
\end{array}\right.
$$

By using the integral representation of Q-function [1]

$$
Q(x)=\frac{1}{\pi} \int_{0}^{\pi / 2} \exp \left(-\frac{x^{2}}{2 \sin ^{2} \theta}\right) d \theta
$$

and averaging (10a) and (10b) over channel distributions, we can further obtain

$$
\left\{\begin{array}{l}
\operatorname{Pr}(\mathbf{x} \rightarrow-\mathbf{x}) \stackrel{\Gamma \rightarrow \infty}{\approx} \frac{3}{16 \lambda_{1 d} \lambda_{2 d}} \Gamma^{-2} \\
\operatorname{Pr}\left(\mathbf{x} \rightarrow\left(-x_{1}, x_{2}\right) \mid \Phi_{o f f}\right) \stackrel{\Gamma \rightarrow^{\infty}}{\approx^{\infty}} \frac{1}{4 \lambda_{1 d}} \Gamma^{-1}
\end{array}\right.
$$

It is observed that the error event that both of the two source symbols are flipped at the receiver has the same conditional probability under any relaying status as shown in (10a), and the corresponding diversity order is always 2 . Therefore, the dominant error event occurs when only one of the source symbols is flipped at the receiver, which determines the overall diversity performance. As the power scaling coefficient $\alpha$ and the combining weight $w$ may depend on channel conditions too, we shall derive the unconditional PEPs of (10c) and (10d) in later sections when discussing the detailed scheme design.

\footnotetext{
${ }^{2}$ The symbol $h$ means the probability is conditional on the related channels. Same convention is used throughout this work.
}

\section{RELAY-SIDE SCHEMES}

In this section, we shall develop two power scaling schemes at the relay side. For both methods, the combining weight $w$ in (6) is set to 1, i.e., the regular MDD with equal weights is employed at the receiver. We demonstrate that full diversity can be achieved by smartly designing the power scaling coefficient $\alpha$ according to channel conditions.

\section{A. Link Adaptive Relaying (LAR)}

LAR was first proposed in [8] for the repetition-coding based DF protocol. The idea is to adapt the relay power to the source-relay-destination channel conditions so as to limit the interference of relay decoding error. However, LAR cannot be employed directly in the network-coded system, where there are multiple source-relay channels instead of a single one as considered in [8].

To extend the spirit of LAR, we first develop a virtual channel model for the relay branch. For the real link, the relay node simply forwards an estimate $x_{r, \oplus}$ of $x_{\oplus}$ to the destination, which provides side information of source symbols $x_{1}$ and $x_{2}$. As we have seen, the diversity order depends largely on the reliability of $x_{r, \oplus}$. Suppose now the destination just decodes $x_{\oplus}$ as $x_{d, \oplus}$ based on the observation $y_{r, d}$, then the end-to-end BER $\operatorname{Pr}\left(x_{d, \oplus} \neq x_{\oplus}\right)$ is a good measure of the reliability of this two-hop relay branch. To this end, we first approximate the conditional error rate at the relay node as

$$
\begin{aligned}
& \operatorname{Pr}\left(x_{r, \oplus} \neq x_{\oplus} \mid h\right)=Q\left(\sqrt{2 \gamma_{1 r}}\right)+Q\left(\sqrt{2 \gamma_{2 r}}\right) \\
& -2 Q\left(\sqrt{2 \gamma_{1 r}}\right) Q\left(\sqrt{2 \gamma_{2 r}}\right) \approx Q\left(\sqrt{2 \gamma_{s r, \text { min }}}\right)
\end{aligned}
$$

where $\gamma_{s r, \text { min }}=\min \left(\gamma_{1 r}, \gamma_{2 r}\right)$ represents the SNR of the weaker source-relay channel. As $\gamma_{1 r}$ and $\gamma_{2 r}$ are independent exponential random variables, $\gamma_{s r, \min }$ is also an exponential random variable with mean $\Gamma_{s r, \min }=\lambda_{s r, \min } \Gamma$, where $\lambda_{s r, \min }=\frac{\lambda_{1 r} \lambda_{2 r}}{\lambda_{1 r}+\lambda_{2 r}}$. The approximation in (13) is quite tight when both of $\gamma_{1 r}$ and $\gamma_{2 r}$ and their difference are reasonably large, as the Q-function $Q(x)$ decays really fast with $x$. The above approximation shows that the multipleinput single-output source-relay channel can be accurately characterized by a single-input single-output virtual channel with the channel input being the XORed source message $x_{\oplus}$ and the channel SNR being $\gamma_{s r, m i n}$, since the conditional BER $\operatorname{Pr}\left(x_{r, \oplus} \neq x_{\oplus} \mid h\right)$ over the virtual channel, which happens to be $Q\left(\sqrt{2 \gamma_{s r, \min }}\right)$, is approximately the same as that over the real channel.

Similarly, the end-to-end BER of the two-hop relay branch can be approximated as

$$
\operatorname{Pr}\left(x_{d, \oplus} \neq x_{\oplus} \mid h\right) \approx Q\left(\sqrt{2 \gamma_{s r d, v}}\right),
$$

where $\gamma_{s r d, v}=\min \left(\gamma_{s r, m i n}, \alpha \gamma_{r d}\right)$. Using the same arguments, we can further model this two-hop branch as a point-to-point virtual channel with the channel input being $x_{\oplus}$ and the equivalent channel SNR being $\gamma_{s r d, v}$. Clearly, when $\gamma_{s r, \text { min }} \leq \gamma_{r d}$, the source-relay channel becomes the bottleneck, so increasing $\alpha$ beyond $\frac{\gamma_{s r, m i n}}{\gamma_{r d}}$ makes no sense 
as $\gamma_{s r d, v} \equiv \gamma_{s r, \text { min }}$. On the other hand, if $\gamma_{s r, \min } \geq \gamma_{r d}$, then the relay-destination channel becomes the bottleneck and the relay node should forward the message with full power. With the above observation, we can design the power scaling coefficient $\alpha$ as

$$
\alpha=\left\{\begin{array}{l}
\min \left(\frac{\gamma_{s r, m i n}}{\gamma_{r d}}, 1\right), \text { global CSI } \\
\min \left(\frac{\gamma_{s r, m i n}}{\Gamma_{r d}}, 1\right), \text { local CSI }
\end{array}\right.
$$

In some sense, the relay node behaves like the system coordinator that is seeking to balance the channel SNRs of the two hops. Finally, we have the following proposition.

Proposition 1: Both of the global CSI based LAR and local CSI based LAR can achieve a diversity order of 2 .

Proof: See [15].

\section{B. ON-OFF Relaying}

Different from soft power scaling in LAR, the relay node could instead apply hard power scaling (i.e., $\alpha \in\{0,1\}$ ). Such ON-OFF relaying has been deeply discussed in [6]-[7] for the repetition-coding based DF system.

As for the network-coded uplink, we propose to turn on the relay node (i.e., $\alpha=1$ ) when

$$
\sum_{\hat{\mathbf{x}} \neq \mathbf{x}} \operatorname{Pr}\left(\mathbf{x} \rightarrow \hat{\mathbf{x}} \mid \Phi_{o n}, h_{1 r}, h_{2 r}\right) \leq \sum_{\hat{\mathbf{x}} \neq \mathbf{x}} \operatorname{Pr}\left(\mathbf{x} \rightarrow \hat{\mathbf{x}} \mid \Phi_{o f f}\right) .
$$

That is, the relay node always chooses the action that promises the smaller total PEP based on local CSI. If (16) is false otherwise, the relay node should stay idle by letting $\alpha=0$. However, there is no closed-form expression of the exact decision rule, as the average of Q-function over channel distribution is hard to manipulate. Alternatively, we can use the high-SNR approximations

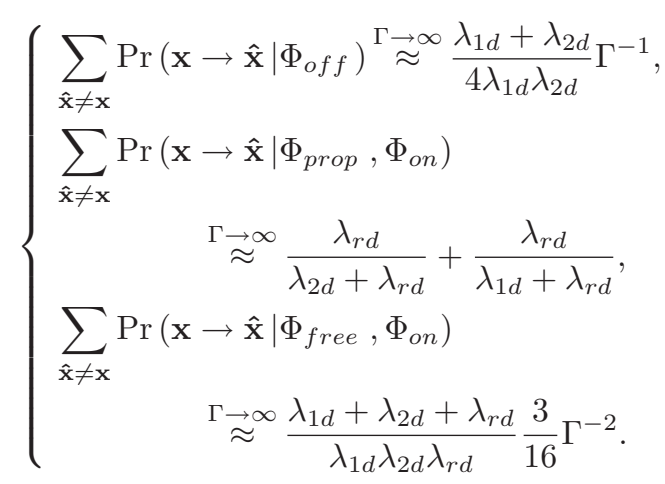

to simplify (16) as

$$
\gamma_{s r, \min } \geq \log \lambda_{T} \Gamma
$$

where

$$
\lambda_{T}=\frac{2 \lambda_{1 d} \lambda_{2 d} \lambda_{r d}\left(\lambda_{1 d}+\lambda_{2 d}+2 \lambda_{r d}\right)}{\left(\lambda_{1 d}+\lambda_{2 d}\right)\left(\lambda_{1 d}+\lambda_{r d}\right)\left(\lambda_{2 d}+\lambda_{r d}\right)}
$$

is a constant determined by the second-order statistics. Consequently, the complex decision rule (16) is simplified to the threshold-based relaying strategy. We observe that the two source-relay channels have to meet the same SNR threshold, as the relay decoding error is bounded by the weaker channel as shown in (13). It is also observed that imposing any threshold having the form of $\log \left(\lambda_{T, k} \Gamma\right)$ on $\gamma_{k}$ for some constant $\lambda_{T, k}>0$ and $k=1,2$ would lead to the same diversity performance, since $\log \lambda_{T, k} \Gamma \stackrel{\Gamma \underset{\approx}{\approx}}{\approx} \log \Gamma$. The special $\lambda_{T}$ given in (19) can be justified by the following proposition.

Proposition 2: For all the ON-OFF relaying protocols with $\Phi_{\text {on }}=\left\{\gamma_{k r} \geq \log \lambda_{T, k} \Gamma, k=1,2\right\}$, where $\lambda_{T, k}$ is any positive constant, a diversity order of 2 can be achieved. Besides, $\lambda_{T, 1}=\lambda_{T, 2}=\lambda_{T}$ is optimum in the sense of minimizing the total end-to-end PEP.

$$
\text { Proof: See [15]. }
$$

\section{RECEIVER-SIDE SCHEMES}

Indeed, the error propagation issue could also be addressed at the receiver side when there is no power scaling at the relay side. So in this section, we shall develop two smart detection schemes at the destination that can also recover the diversity loss. For both methods, we assume that the relay node always forwards message using full power (i.e., $\alpha=1$ ).

\section{A. Link Adaptive Combining (LAC)}

In LAR and ON-OFF relaying, the destination implicitly assume there is no relay decoding error, thus the combining weight $w$ is always set to 1 . Basically, the combining weight is a kind of confidence measure that reflects how reliable the relay link is. When the relay decoding error occurs with high probability, the destination should adaptively lower the combining weight to discount the contribution of the relay branch in (6). We note that similar idea has been adopted in [9] for the repetition-coding based DF protocol.

Before describing our adaptive design of $w$, let us first revisit the virtual source-relay-destination channel. As mentioned before, this virtual channel has the BPSK input $x_{\oplus}$ and the channel SNR $\gamma_{s r d, v}$. As the relay-destination channel coefficient is $h_{r d}$, we can approximate the real received signal $y_{r d}$ in (5) as

$$
\tilde{y}_{r d}=\bar{h}_{r d} \sqrt{\alpha} x_{\oplus}+\tilde{n}_{r d}
$$

where $\tilde{n}_{r d} \sim \mathcal{C N}\left(0, \frac{\left|\bar{h}_{r d}\right|^{2} \alpha}{\gamma_{s r d, v}}\right)$ is the virtual additive noise, and the noise power is such that the SNR of this virtual signal is exactly $\gamma_{s r d, v}$. With the virtual signal model, the MLD given the observations $y_{1 d}, y_{2 d}$ and $\tilde{y}_{r d}$ is

$$
\begin{aligned}
\mathbf{x}_{d}=\arg \max _{\hat{x}_{1}, \hat{x}_{2} \in \Omega} g & \left(\tilde{y}_{r d}-\bar{h}_{r d} \sqrt{\alpha} \hat{x}_{\oplus},\left|\bar{h}_{r d}\right|^{2} \alpha / \gamma_{s r d, v}\right) \\
& \times \prod_{k \in\{1,2\}} g\left(y_{k d}-\bar{h}_{k d} \hat{x}_{k}, N_{0}\right),
\end{aligned}
$$

where we exploit the independence of the three received signals. After some manipulations, we can show that the above MLD is equivalent to the weighted MDD in (6) by letting

$$
w=\frac{\gamma_{s r d, v}}{\gamma_{r d}}=\min \left(\frac{\gamma_{s r, \min }}{\gamma_{r d}}, 1\right) .
$$


Clearly, this adaptive weight is always less than or equal to 1. When $\gamma_{s r, \min } \leq \gamma_{r d}$, i.e., the bottleneck is the sourcerelay channel, the destination should discount the contribution of the relay branch as the incoming signal is unreliable. On the other hand if $\gamma_{s r \text { min }}>\gamma_{r d}$, the relay-destination channel becomes the bottleneck, then the source-relay channel is given full credit as the other two source-destination channels. We remark that the above weight design is based on global CSI. When $\gamma_{s r, \min }$ is unavailable at the destination, we could apply its average instead, i.e.,

$$
w=\min \left(\frac{\Gamma_{s r, \min }}{\gamma_{r d}}, 1\right) .
$$

By comparing global CSI based LAR (15a) and global CSI based LAC scheme (22), we observe that

$$
\alpha w \gamma_{r d}=\gamma_{s r d, v}=\min \left(\gamma_{s r, \min }, \gamma_{r d}\right) \triangleq \gamma_{s r d, \min } .
$$

This factor can be regarded as the aggregate scaling coefficient imposed on $x_{r, \oplus}$ to account for the relay decoding error. So the two schemes are actually following the same principle to address the error propagation issue, and this design goal is fulfilled either at the relay side (i.e., LAR) or at the receiver side (i.e., LAC). Due to such relations, one may guess that both of the global CSI based LAC (22) and local CSI based LAC (23) can achieve a diversity order of 2 as is the case of LAR schemes. However, this is not true as we show in the proposition below.

Proposition 3: For the global CSI based LAC scheme (22), a diversity order of 2 can be achieved; however, the diversity order of the local CSI based LAC scheme (23) is only 1 .

Proof: See [15].

\section{B. Maximum Likelihood Detection (MLD)}

So far, we have assumed that the linear combiner is employed at the destination; however, full diversity cannot be achieved when the receiver only knows local CSI. So in this subsection, we study the diversity performances of MLD, which is optimum in the sense of minimizing detection errors.

The MLD based on real observations $y_{1 d}, y_{2 d}$ and $y_{r d}$ can be expressed as

$$
\begin{aligned}
\mathbf{x}_{d} \triangleq \arg \max _{\hat{x}_{1}, \hat{x}_{2} \in \Omega} f & \left(y_{r d} \mid \hat{x}_{1}, \hat{x}_{2}\right) \\
& \times \prod_{k \in\{1,2\}} g\left(y_{k d}-\bar{h}_{k d} \hat{x}_{k}, N_{0}\right)
\end{aligned}
$$

where

$$
\begin{aligned}
f\left(y_{r d} \mid x_{1}, x_{2}\right)= & g\left(y_{r d}+\bar{h}_{r d} x_{\oplus}, N_{0}\right) \operatorname{Pr}\left(\Phi_{\text {prop }}\right) \\
& +g\left(y_{r d}-\bar{h}_{r d} x_{\oplus}, N_{0}\right) \operatorname{Pr}\left(\Phi_{\text {free }}\right),
\end{aligned}
$$

is the conditional PDF of $y_{r d}$ given the two source symbols $x_{1}$ and $x_{2}$, and (13) and (9) should be plugged in the term $\operatorname{Pr}\left(\Phi_{\text {prop }}\right)$ for global CSI based MLD and local CSI based MLD, respectively. Our main result is summarized below.

Proposition 4: Both of the global CSI based MLD and local CSI based MLD can achieve a diversity order of 2 .

Proof: See [15].

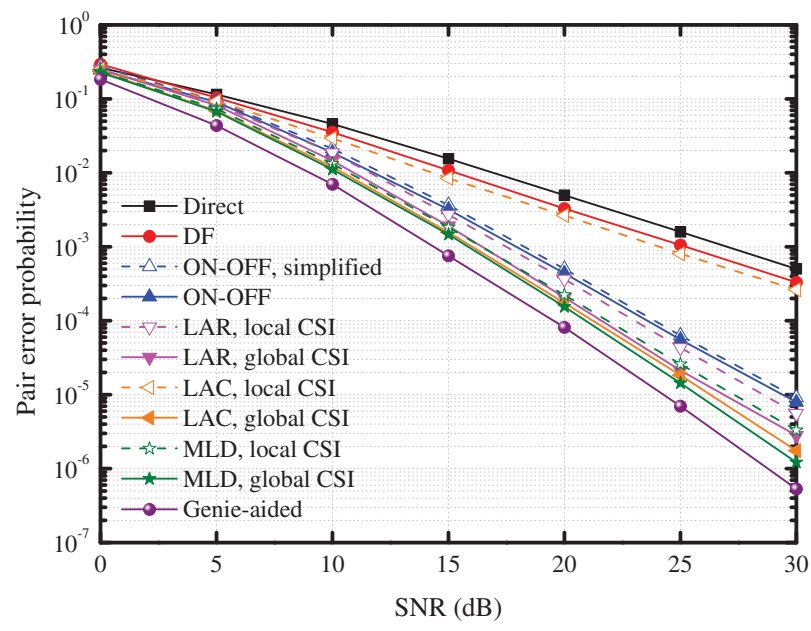

Fig. 1. Error performances of BPSK signal in a symmetric network.

\section{SIMULATIONS}

In this section, we present some simulation results to validate our diversity analysis. Throughout our simulations, we use the path loss model $\lambda=D^{-3}$, where $\lambda$ is the channel gain and $D$ is the distance between two terminals. Pair error probability is used as the performance metric, i.e., the probability that at least one of the source symbols is decoded incorrectly at the destination. BPSK signal is used throughout simulations.

Fig. 1 shows the error performances in a symmetric network, where all the distances are normalized. We observe that local CSI based LAC only achieves a diversity order of 1 as direct transmission and simple DF without error control, while all the other schemes achieve a diversity order of 2 . The genie-aided relaying with perfect error detection is the benchmark for all the practical schemes, thus having the best error performances. It can also be seen that the performance of the simplified ONOFF relaying (18) is very close to that of the exact decision rule (16) at all SNRs. Local CSI based LAR is slightly better than the ON-OFF relaying through soft power scaling. The performances of the three global CSI based methods are very close. Comparatively, MLD is the best scheme among all by explicitly taking into account the relay decoding error, but the performance is nearly the same as the performance of LAC, which enjoys lower decoding complexity. By comparing global CSI based LAR and MLD with their local CSI based counterparts, about $1.3 \mathrm{~dB}$ SNR gain is observed at a cost of higher signalling overhead.

Next we investigate the error performances with different relay positions. For the network topology, we place the destination at $(0,0)$, and locate the two source nodes at $\left(\frac{\sqrt{3}}{2}, \pm \frac{1}{2}\right)$, respectively. The relay node shall move along the $\mathrm{x}$-axis from $(0.2,0)$ to $(2,0)$. The error performance is shown in Fig. 2. It is observed that for all the schemes, the best performance is attained when the relay node is close to the source nodes, as the relay decoding error dominates the overall system performance. In all cases, global CSI based schemes perform much better than their local CSI based counterparts at a cost 


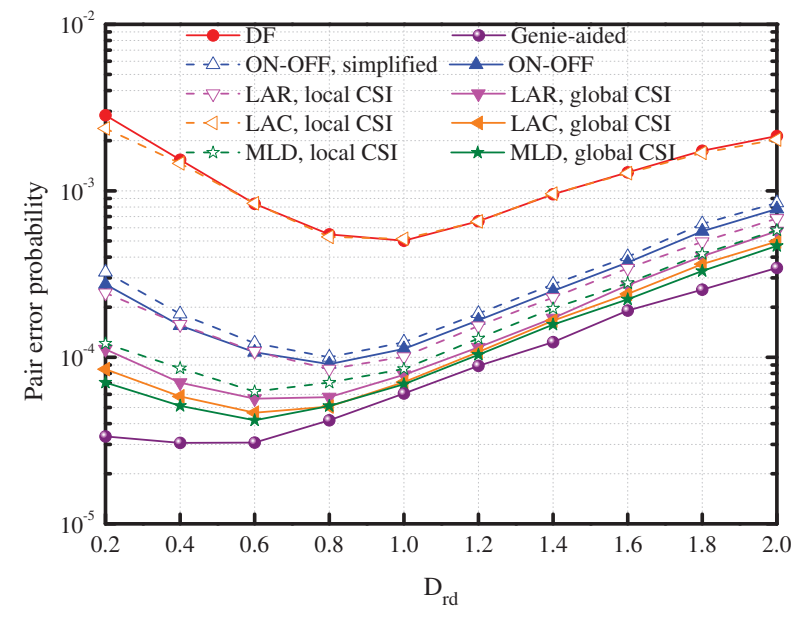

Fig. 2. Error performances of BPSK signal with $\Gamma=20 d B$ and different relay positions.

of higher signalling overhead.

As the relay node only uses a portion of its total power in the relay-side schemes, we also plot the relay power consumption ratio in Fig. 3. It is observed that the relay power consumption of ON-OFF relaying increases with SNR, while for LAR it is independent of SNR because the relay power depends only on the relative quality of source-relay channel and relay-destination channel. For LAR, we observe that the power consumption is really low when the relay is close to the destination and the source-relay link is comparatively unreliable; as the relay node moves far away from the destination, the relay node gradually increases its power until the relay-destination channel becomes the bottleneck. For $\mathrm{ON}-\mathrm{OFF}$ relaying and genie-aided relaying, the relay power consumption maximizes when the relay node is close to the source, in which case the relay decoding is really reliable and the chance of forwarding the message is high. Note that although the relay node always uses full power in the receiverside schemes, better performances can be achieved compared to the relay-side schemes. So there is a basic tradeoff between error performances and power consumption.

\section{CONCLUSIONS AND FUTURE WORK}

In this work, we have developed two power scaling schemes at the relay side and two detection schemes at the receiver side, respectively, that can mitigate error propagation and thus achieve full diversity in the wireless network-coded uplink. We also set up a virtual channel model to facilitate the analysis of end-to-end error performance. We remark that the error propagation issue is addressed either at the relay side or at the receiver side in this work to achieve full diversity. One interesting issue for possible future consideration is how to jointly optimize the relaying scheme and detection scheme so as to further improve the coding gain.

\section{REFERENCES}

[1] K. J. R. Liu, A. K. Sadek, W. Su, and A. Kwasinski, Cooperative Communications and Networking, Cambridge University Press, 2008.

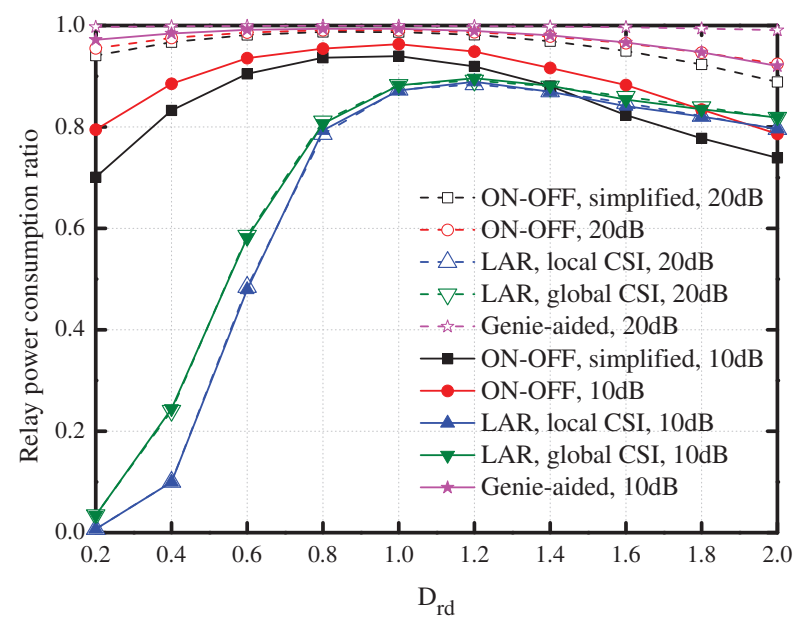

Fig. 3. Relay power consumption ratio with different relay positions.

[2] J. N. Laneman, D. N. C. Tse, and G. W. Wornell, "Cooperative Diversity in Wireless Networks: Efficient Protocols and Outage Behavior," IEEE Trans. Inf. Theory, vol. 50, no. 12, pp. 3062-3080, Dec. 2004.

[3] W. Guan and K. J. R. Liu, "Performance Analysis of Two-Way Relaying with Non-Coherent Differential Modulation," IEEE Trans. Wireless Commun., vol. 10, no. 6, pp. 2004-2014, June 2011.

[4] W. Guan and K. J. R. Liu, "Two-Way Denoise-And-Forward Relaying With Non-Coherent Differential Modulation," in Proc. IEEE Global Telecommun. Conf., Dec. 2011.

[5] M. Xiao and M. Skoglund, "Multiple-User Cooperative Communications Based on Linear Network Coding", IEEE Trans. Commun., vol. 58, no. 12, pp. 3345-3351, Dec. 2010.

[6] F. A. Onat, A. Adinoyi, Y. Fan, H. Yanikomeroglu, J. Thompson, and I. Marsland, "Threshold selection for SNR-based selective digital relaying in cooperative wireless networks," IEEE Trans. Wireless Commun., vol. 7, no. 11, pp. 4226-4237, Nov. 2008.

[7] T. Himsoon, W. P. Siriwongpairat, W. F. Su, and K. J. R. Liu, "Differential Modulation With Threshold-Based Decision Combining for Cooperative Communications," IEEE Trans. Signal Process., vol. 55, no. 7, pp. 3905-3923, July 2007.

[8] T. Wang, G. B. Giannakis, and R. Q. Wang, "Smart Regenerative Relays for Link-Adaptive Cooperative Communications," IEEE Trans. Commun., vol. 56, no. 11, pp. 1950-1960, Nov. 2008.

[9] T. Wang, A. Cano, G. B. Giannakis, and J. N. Laneman, "High Performance Cooperative Demodulation With Decode-and-Forward Relays," IEEE Trans. Commun., vol. 55, no. 7, pp. 1427-1438, July 2007.

[10] M. D. Selvaraj, R. K. Mallik, and R, Goel, "Optimum Receiver Performance With Binary Phase-Shift Keying for Decode-and-Forward Relaying," IEEE Trans. Veh. Technol., vol. 60, no. 4, pp. 1948-1953, May 2011 .

[11] A. Nasri, R. Schober, and M. Uysal, "Error Rate Performance of Network-Coded Cooperative Diversity Systems," in Proc. IEEE Global Telecommun. Conf., Dec. 2010.

[12] M. Iezzi, M. D. Renzo, F. Graziosi, "Closed-Form Error Probability of Network-Coded Cooperative Wireless Networks with Channel-Aware Detectors," in Proc. IEEE Global Telecommun. Conf., Dec. 2011.

[13] M. Xiao and T. Aulin, "Optimal Decoding and Performance Analysis of a Noisy Channel Network with Network Coding," IEEE Trans. Commun., vol. 57, no. 5, pp. 1402-1412, May 2009.

[14] Z. Han, X. Zhang, and H. V. Poor, "Cooperative Transmission Protocols with High Spectral Efficiency and High Diversity Order using Multiuser Detection and Network Coding," IEEE Trans. Wireless Commun., vol. 8 , No. 5, pp. 2352-2361, May 2009.

[15] W. Guan and K. J. R. Liu, "On The Design of Relaying and Detection Schemes to Mitigate Error Propagation with Wireless Network Coding," submitted to IEEE Trans. Wireless Commun., Nov. 2011. 